\title{
Internationally educated nursing students' experiences of integration in the hospital setting
}

\author{
Yolanda Babenko-Mould ${ }^{* 1}$, Janice Elliott ${ }^{2}$ \\ ${ }^{1}$ Arthur Labatt Family School of Nursing, Faculty of Health Sciences, Western University, London, Canada \\ ${ }^{2}$ Faculty of Human Services, Health Sciences, Nursing, Fanshawe College, London, Canada
}

Received: April 24, 2015

DOI: $10.5430 /$ jnep.v5n9p100
Accepted: May 18, 2015

Online Published: June 28, 2015

\begin{abstract}
In North America, internationally educated nurses (IEN) have played an essential role in addressing the nursing shortage as a result of immigration and increasing international recruitment. Given the importance of the IEN role in the delivery of patient care, it is vital that IENs who are involved in educational programs to prepare them for practice in North America are integrated as health care team members. The purpose of this paper is to explore internationally educated nursing students' experiences of integration in the acute care hospital setting. A qualitative design was used to explore nine IEN students' experiences of integration in the acute care practice setting. IEN students involved in a bridging educational program in nursing participated in individual interviews lasting 60-90 minutes. Interviews were transcribed verbatim. Researchers engaged in meaning making where initial categories were shaped into themes. Participants expressed having a dual identity as nurse and student, feeling like outsiders and experiencing discrimination in the practice setting, and IENs experienced challenges around discontinuity of relationships, language, and use of technology. IENs also discovered opportunities to learn and grow. To support the meaningful integration of IENs into clinical practice, it is crucial that the academic environment and practice partners ensure IEN students have positive and effective learning experiences where they feel part of the interprofessional team, and can ultimately deliver safe client care.
\end{abstract}

Key Words: Internationally educated nurses, Integration, Acute care practice, Healthy teaching and learning settings, Qualitative methods

\section{INTRODUCTION}

Nursing shortages continue to be an issue globally. Within Canada there are predictions for an estimated shortage of 60,000 full time equivalent RNs by $2022 \cdot{ }^{[1]}$ As unemployment rates are predicted to drop in the United States by 2015, nursing shortages thought to be reduced may re-occur. ${ }^{[2]}$ In the United Kingdom, there has been a net outflow of nurses and decrease in funding for nursing education which has led to a shortage of nurses, while international recruitment of nurses doubled from 2010 to 2012. ${ }^{[3]}$ In Western coun- tries, internationally educated nurses (IEN) have played an essential role in addressing the nursing shortage as a result of immigration and increasing international recruitment. ${ }^{[4]}$

Despite the consistent numbers of IENs entering the Canadian nursing workforce, many IENs continue to face personal challenges and structural barriers as they strive to integrate into clinical settings. ${ }^{[5]}$ Overcoming language barriers and cultural differences have been identified as creating feelings of isolation for IENs as they integrate into the workplace. ${ }^{[5]}$ Cultural differences include different role expectations of the

\footnotetext{
*Correspondence: Yolanda Babenko-Mould; Email: ybabenko@uwo.ca; Address: Arthur Labatt Family School of Nursing, Faculty of Health Sciences, Western University, London, Canada.
} 
nurse and differences in health care systems, while structural barriers such as marginalization, racism and discrimination have been reported. ${ }^{[6]}$ Perceived preparatory differences in scope of practice, and communication issues have also been highlighted as challenges that IEN students uniquely experience. ${ }^{[7]}$

In spite of the identified challenges, there remains insufficient system supports to facilitate IENs entering the workforce in Ontario. ${ }^{[8]}$ For IENs who have successfully completed the national regulatory exam, often other roadblocks are encountered when trying to find employment, such as finding an employer willing to hire an IEN. IENs often took a less satisfying job (i.e., personal support workers) where the full extent of their skills was not recognized. ${ }^{[9]}$ When IENs confronted such challenges while attempting to integrate into clinical practice, the outcome has been decreased retention of IENs in the workplace. ${ }^{[10]}$ IENs lacked confidence, felt stigmatized as "refresher nurses", and felt like outsiders as a consequence of the treatment they received from employers and colleagues. ${ }^{[11]}$

In Canada, there are 23,176 internationally educated registered nurses and 2400 internationally educated licensed practical nurses/registered practical nurses with $48 \%$ of the internationally educated registered nurses in Canada working in Ontario. ${ }^{[12]}$ In 2013, IENs made up $29.7 \%$ of new Registered Nurse applicants and $40 \%$ of new Registered Practical Nurse (RPN) applicants in Ontario. ${ }^{[13]}$ While overall numbers of IENs in Canada has remained relatively constant from $7.9 \%$ in 2007 to $8.6 \%$ in $2011{ }^{[12]}$ applications for immigration among female registered nurses as the principal economic applicant has increased from 170 in 2002 to 2150 in 2011. This dramatic increase as principal economic applicant or major breadwinner for the family emphasizes the role of the IEN in supporting the family.

Notwithstanding the previously noted challenges, there remains a paucity of research focused on designing and evaluating interventions to ease IEN's transition into the workplace, the dynamics of cross cultural workforces, and investigation into the perspectives of IENs on nursing care and the role of the nurse - all areas in need of urgent attention according to research scholars focused on nurse retention. ${ }^{[8]}$ Successful transition of internationally educated health professionals to employment is imperative for retention, and ultimately, for quality patient care when there is a shortage of health professionals to meet the increasing needs of society in the Canadian health care system. ${ }^{[9]}$ There is a paucity of literature focused on the experience of IENs as nursing students, as the majority of the literature regarding IENs is situated from a practicing nurse perspective. Therefore, in order to understand IEN students' integration into the acute care setting during a transitional bridging program, the authors conducted a study to explore IENs' experiences in a student role in the acute care practice environment.

The purpose of the study was to explore IEN students' experiences of integrating into an acute care teaching hospital. The primary research question that guided this study was: What are IEN students' perceptions of integration in acute care clinical settings during involvement in a 'Bridging for Internationally Educated Nurses' program (BIEN) program? The aims were: 1) to explore the perceptions of integration of IEN students in the acute care clinical setting, and 2) to understand the needs of IENs for successful integration into an acute care setting. The concept of integration is characterized primarily as a process of combination where two or more elements are merged with sufficient interaction so that unity of the newly formed entity is achieved. The attributes of the concept of integration, therefore, include process, combination, interaction, and unity. ${ }^{[14]}$ In this study, the term integration was conceptualized to mean being accepted as part of a clinical team in a hospital setting where IEN students could learn effectively. In this sense, integration was understood as a process, which involved health professionals working together as one group while maintaining one's own individual style as a nurse.

\section{METHOD}

The research methodology used in this study was hermeneutic phenomenology. Hermeneutic phenomenology has both descriptive (phenomenological) and interpretive (hermeneutic) elements. ${ }^{[15,16]}$ The objective of phenomenology is to fully describe the lived experience with the intent to "let things speak for themselves"; however, hermeneutic phenomenology claims there are no such things as uninterpreted phenomena-even the facts of lived experience need to be captured in language and this is an interpretive (hermeneutic) process. ${ }^{[16]}$ The lived experience is thus described with the intent to understand what that experience meant to those who lived it rather than provide causal explanations of that experience. ${ }^{[16-18]}$ Hermeneutic phenomenology was selected as a methodology for this study to provide for a deeper understanding of IENs' experiences in the acute care setting. Hermeneutic phenomenology as a methodology provides attention to the language and meaning of IENs' experiences. "Phenomenology is the study of the lifeworld-the world as it is immediately experienced rather than as we conceptualize, categorize, or theorize about it" (p. 37). ${ }^{[16]}$ Phenomenology is the search for what it means to be human and for this study to have a fuller grasp of what it means to be an internationally educated nursing student. 


\subsection{Research design}

A qualitative design was used to explore IEN students' experiences of integration in the acute care practice setting. Interpretive phenomenological studies use small sample sizes with the aim of the study to express the perceptions and understandings of the participants. ${ }^{[19]}$ The goal is to develop a rich description of the phenomena.

Phenomenological themes are the structures of experience. A thematic phrase only serves to point at, to allude to, or to hint at, an aspect of the phenomenon. In this study, the highlighting approach was used to uncover themes. In the highlighting approach the researchers read the text several times and asked, "What are the statements or phrases that seem particularly essential or revealing about the experience being described?" (p. 61). ${ }^{[20]}$ During the analysis process, the researchers spent a prolonged period of time independently immersed in the data-reading and re-reading the transcripts and reflecting on the content until an intuitive understanding or crystallization of the text was achieved. When there were differences between researchers in identifying meaning from the content, further discussion occurred until consensus was negotiated among the researchers. Themes representing descriptive categories formed a preliminary framework for analysis, such as words, phrases or events that appeared to be similar were grouped into the same category. These categories were gradually modified or replaced during the subsequent stages of analysis that followed.

\subsection{Sample}

After obtaining institutional ethics approval, twenty IEN students who had been enrolled in a "bridging program" were contacted. The bridging program offered at the community college provided IENs, both Registered Nurse and Registered Practical Nurse, educational preparation to meet competency standards for safe practice in Ontario. The program offers the IEN an individualized program completed on a part-time basis and includes both classroom and clinical experiences. IEN students who had completed an acute care clinical course in the bridging program were invited to participate in this study. The final sample consisted of 9 IEN students ( $8 \mathrm{fe}-$ males and 1 male) who had completed an acute care 200 hour clinical placement experience on either a medical or surgical unit.

\subsection{Sampling procedures}

The participants were purposefully selected for this study based on their first-hand experience with the phenomena of interest (student perceptions of integration in the acute care practice setting). Internationally educated nursing students were invited to participate through recruitment activi- ties which included posters, class announcements, and postings in the college program online website.

Data for this study were collected during individual interviews with IEN students that were 60-90 minutes in length. All students enrolled in the bridging IEN program were informed about the study and invited to participate in an interview. Letters of information were provided to all IENs in the program. At the beginning of the interview, each participant received a letter of information about the study, questions about the study were addressed, and then written consent to participate was obtained from each IEN student. The interview facilitators reminded participants that only themes emerging from the data would be reported and that participants' names would be replaced by pseudonyms when direct quotes were being used. Interviews were conducted by members of the research team who were not involved in the teaching or supervision of any IEN students in either theory or clinical practicum experiences. A semi-structured interview guide was used during the interview process to invite each participant to share their perceptions of integration in an acute care practice setting during their involvement in a bridging program for IEN students. Each interview was audio recorded and then transcribed verbatim.

\section{RESULTS}

The average age of participants was 40. Although both male and female IEN students were invited to participate in the study, most participants were female and their first language included Spanish, Arabic, or Tagalog. Prior to immigrating to Canada, the IENs reported an average of 9 years of nursing experience in varied practice settings, including the operating room, medical-surgical units, the emergency room, and intensive care units. The IENs in this study had lived in Canada for an average of 7.5 years (range of 3-8 years) prior to initiating an application for registration as a nurse in Canada. Prior to their enrollment in the bridging program for IENs, some participants had been employed in Canada as personal support workers or as registered practical nurses.

There were 5 main themes identified during data analysis related to IENs' experiences of integration in an acute care practice environment: (1) Dual identity - that of a nurse and of a student; (2) An insider some of the time, an outsider most of the time; (3) Comparing and contrasting health care experiences; (4) Challenges of Dis-continuity, Communication and Technology; and (5) Finding opportunities to learn and grow.

In the theme Dual identity- that of a nurse and of a student, the IENs felt that their past nursing experience went largely unacknowledged by patients, administrators and staff nurses. 
They wanted to be trusted, and to work with nurses who expressed confidence in their knowledge and skills. Instead, they were often perceived as a first year student without clinical practice experience. As Bethany, a RN shared:

Well, I guess like any new person... you have to gain that confidence... when people will see you... okay that person is new... people tend to see you as being in the [nursing] program. I guess that was my experience that you are a student right. So you aren't quite a nurse, you're a student so a few nurses try to explain to you like okay this is a syringe. I know... that's basic.

Karen, with ten years of experience shared her frustration with not being given enough responsibility: "Working with the nurse, I expect from them to give us an assignment because we are, we are nurse; we're not like a student..."

The IENs wanted to be recognized for their experience and education and that they would be trusted as nurses. Bethany, who had worked in the emergency room, commented about her desire for the co-assigned nurse to recognize her abilities: "You're trying to say okay I know, just see that I know, just let me show you that I can do it." Natasha, who had more than 20 years of experience stated: "I hope for the future RNs like me that they can practice with more hands on... if they give you the trust and the confidence, then they can practice... there's no hesitation." Janelle, a RN with several years of experience in acute care spoke about the importance of the role of the clinical teacher. She felt that the clinical teacher must recognize the IEN as a nurse but also to be responsible for the IENs as students. One aspect of the role a clinical teacher assumes is the preparation of nursing staff in the clinical area to work with the IENs. Michelle felt that the clinical teacher had not prepared the nursing staff very well and that the staff nurses did not know how to work with international nurses.

With respect to the theme of feeling like an insider or outsider, if the IEN was included and felt part of the team, they felt like an insider but often they felt not part of the team or not accepted by the clients and then they felt like an outsider. IEN students experienced feeling like an outsider when patients did not treat them as a professional or rejected their care. Karen shared her concern about being rejected by patients because she was an IEN: "So what is client expecting from me and what he would feel about me as an international, not western one, not from Canada... [Would client] reject me or [he] wouldn't want [me] to touch him?" Natasha, who had experience as a nurse clinician and teacher commented about the lack of respect experienced from the patients: "Some patients are not polite and in my country being a nurse you

Published by Sciedu Press are very respected." Natasha continued:

Maybe it's because we're coloured and we're not from this country, sometimes you underestimate your skills and your ability, then sometimes they (patients) are afraid we cannot handle that right... they're asking us is there any nurse aside from you?

Some nurses, knowingly or not, engaged in making IEN students feel like an outsider by not including them more fully in client care. Natasha commented:

They [nurses] are the ones talking to the patient... we're just like a shadow... They [nurses] think we make a mistake because we're from another country. . . they think maybe we're different. . they think we are not safe to practice... we also do safely practice because in my country if you have one mistake, even a medical drug error once, you lose your license right away so we're very, very careful too.

Another reason for the IENs being integrated into the unit depended on whether the clinical teacher was viewed as an insider or outsider. If the clinical teacher was part of the unit or well known to the staff then it was much easier for the IENs to find nurses that were willing to work with them.

The health care system in the country of origin for each IEN participant was different in many ways compared to the Canadian health care system. The IENs compared and contrasted many areas including: technology, nurse-patient ratios, and the role of the nurse. The role of the nurse differed from the IENs' home country in relation to the role of the nurse in Ontario. There were differences in providing care to both male and female patients. Karen shared the differences in cultures when dealing with male patients:

I was dealing with a male client, in my country is different than here because they keep personal things to male, male nurse only to deal with that... male patients don't let the female nurse touch them... Yeah, it was a challenge like for example in the CCU [critical care unit] I was inserting a male catheter. It was my first time you know.

Differences in the provision of care were noted based on religious or personal background. One IEN commented that there are no cultural, personal, or religious restrictions on provision of patient care and that as a nurse, one cares for everybody. There were differences in the roles of the nurse from the IENs' home country. Karen also commented that 
aspects of the role of the nurse in Ontario would be completed by the doctor in her home country. "Here in Canada, they are looking for teaching clients more, educating more, and like for discharge, very big issue for them to refer, in my country this kind of thing it's the doctor."

Being a member of a health care team with several health care professionals both within nursing and outside nursing was a new experience for some of the IENs. The scope of the nurse's role was much different from their home country. Several of the IENs commented on the importance of learning about discharge planning and the administrative aspects of nursing in Canada. Michelle who had more than twenty years of experience commented:

It wasn't enough to have the clinical because here the administration issues are really different from my country, so nurses have to work not just with patients, they have to work with computers, they have to work to know about the long term care nursing home, they work with a physiotherapist, they work with an occupational therapist - all the people, all people work together. In clinical hours you don't have the opportunity to work with all these kinds of thing.

Janelle spoke about her experiences as a $\mathrm{RN}$ in Columbia. As the RN, she would care for 25 or 30 patients with a personal support worker. The personal support worker had an expanded role where they would be giving medications and performing many skills. When she came to Canada, she was surprised to see the changes in the personal support worker role from Columbia and was especially surprised to learn about the registered practice nurse and the communication clerk role; there was no equivalent in Columbia.

In nursing, continuity of care is an important part of practice. That perspective was not equally valued in the realm of clinical education with students. When an IEN was working with different nurses every day, the IEN would meet with different expectations. Janelle stated that one assigned nurse would want the IEN to have everything checked and the assigned nurse for the next shift would question the IEN's confidence in giving medications when she asked for her medications to be checked. Some of the IENs spoke about the difficulty with getting to know the nurses when they changed frequently. Maxine shared:

The nurses change all the time so it is difficult to know all the nurses... I don't know, it was hard to go every day to ask somebody who wants to work with me... It was difficult to contact the nurse, yeah do you want to work with me, do you understand me?
Patricia stated, "It's not easy to introduce yourself the first day because every shift you will see different people." For Janelle, the change in the nursing staff on a daily basis was very different from her experiences in her home country of Columbia. The nurses would work the same 6 hour shift so there was very little change in staffing based on the shift and if there were casual nurses called in to work, they were always the same nurses. She also commented that the lack of continuity in care in Canada resulted in an increased amount of time required to get information about the client by the nurses.

Some of the IENs spoke about the lack of feeling welcomed on the nursing unit. For example, Bethany commented "Can we be with you?... so it's pretty much you feel like you're begging like please take me." Michelle expressed how difficult it was to develop relationships with the nurses on the unit. "Actually, people start in that way you say good morning and none of the nurses says to you... Other times, sometimes they say hello sometimes they didn't want to speak to you." This perceived lack of support affected the IENs' integration onto the nursing unit.

However, there were some nurses who were welcoming, respected IEN students' needs, and helped them feel like an insider as part of the team. Rachel stated:

From the beginning, we have an orientation and they said if you don't understand just let us know. Most of the nurses they are really good, really good. They welcome us, they encouraged us, they helped us, they teach us everything and they give us information on whatever we ask. . I know sometimes because they are so busy and it's a busy time.

Another IEN commented on the support that she received from the nurses, "If I can't pronounce or I don't understand usually I ask them to, can you just explain to me?" The nurses that were supportive and welcoming of the IEN helped the IEN to be integrated into the nursing unit.

The IENs who felt they were less proficient in English found communication more challenging in the clinical setting. The IENs identified written documentation as a challenge including abbreviations and short forms. One IEN was concerned about her ability to communicate with the patients. Philomene stated:

You have to translate all your profession to another language. So you have to learn how to speak to the patients because at the school you learn from books but in the field like the English is really different, and sometimes when I speak 
to the patients they cannot understand me, they can't, nurses they have, they are patient, so they try to understand me but no the patients have, they are ill, they are ill...

Janelle added that she was nervous when speaking with doctors, and when calling the pharmacy. She found it a challenge when asking someone to spell their name because of the differences in the alphabet from her first language to English. Janelle found communication a challenge with the difference in pronunciation of medications such as Lasix. Lasix is spelled similar in both Canada and Columbia but based on the pronunciation, it was difficult for Janelle to understand the name until she was able to see the name of the medication in writing.

Without the non-verbal cues, some IENs found that communicating by phone or listening to report on tape to be frightening. Janelle summarized her feelings about the phone as "The monster is the phone." Bethany also expressed concern about talking on the phone in an emergency situation. "Oh my god what if I call and ask for the respiratory therapist, they don't understand me and like it's a really acute patient..." Olivia expressed her difficulties with listening to taped report:

After almost six months, tape, listen to the report sometimes I can ask some nurses, they have or they translate or explain me because they use too many abbreviations. And you know the nurses speak in normal language so sometimes I couldn't understand it the tape, this one was hard for me, actually for me the tape is too hard.

Rachel also had difficulty with taped report. "The report, oh most of the time I didn't understand half of it because they say it fast."

Another challenge for IENs was selecting the right word while communicating. Michelle added:

Maybe when you want to say something and you don't know how to say, you try to change the words so maybe that word are not the best word for you, it's not intention to be rude but sometimes I am, I am rude without intention. Yeah for example I say I would like to check, check is supervised, I don't say I would like to see.

Several IENs recognized the need to improve their communication in English through practice with speaking. New or different technologies were identified as a major challenge for some of the IENs. Sarah who had 10 years of experience as a nurse prior to coming to Canada described her challenges with new technology:

Okay the procedures - I checked blood sugar which was a new monitor, we had to go through the exam for that and bladder scanning was also a new procedure for me, and most of the things were new because they were very modern like the ceiling lift... the bath chair, beds how to operate the beds and everything, and the most important thing is the monitors; the IV pump... IV pump is still very tricky. We are still learning.

Another area in technology that was identified as a challenge was the use of the computers. Rachel commented, "we use the computer inside the ward too because it's something I never heard about it in the clinical practice."

IENs who felt they had stronger skills in English seemed to be more apt to seek out additional learning opportunities. They expressed feeling more confident to do so over their colleagues who struggled with communication. Some IENs would take every opportunity to increase their knowledge. Michelle was very keen to get every experience possible: "Tried to use every opportunity that I had to learn because I like the hospital, I work in the hospital too many times in my country, so I tried to, to spend my time as helpful as possible, as useful as possible for me." Melissa was content with her experiences working with the health care team. "I have the opportunity to work with great professionals that gave me the opportunity to learn from them and support me within the practice."

Several IENs felt that there should have been more emphasis in orientation about policies and procedures. Through understanding the policies and procedures ahead of time, one IEN felt that she could have completed simple procedures when asked by the co-assigned nurse. Janelle stated: "Protocols and procedures that I told my teacher because I was curious and I was looking for information, information because I know that if I have information I feel more confident then in practice."

Another IEN expressed her desire for further education and to increase her knowledge: "And another thing that is really important, we don't know all the stuff here about nurse so at this moment, I want to continue studying because I know it's really important to continue to do some courses to get certificates."

\section{Discussion}

Based on the findings, it is apparent that a number of opportunities could be developed or enhanced to support IEN 
integration into the clinical setting. Similar to a study conducted by Higginbottom, ${ }^{[21]}$ IENs in this study stated that their expectations with respect to a professional role were unmet and that they felt disillusioned about the regulatory requirements to practice as RNs and the ability for them to practice in the specific area of their expertise. IENs living in Canada were not being integrated into the workforce as effectively as they could be. Experiencing a dual identity as a nurse and student was difficult for IENs. The IENs wanted to be recognized as being experienced and knowledgeable qualified nurses while also having the opportunity to learn as a student. Each IEN had unique needs which required a more individualized approach from nurses in the clinical area and clinical faculty to facilitate IENs' learning. If IENs' experiences and knowledge continue to be perceived as being devalued, then a feeling of silencing and disenfranchisement could occur, which could contribute to attrition of IEN students from BIEN programs.

To enhance understanding of experiences of IENs, there should be increased education for nursing staff and administration about the varying strengths and challenges experienced by IENs. Pre-arrival orientation and structured and comprehensive workplace orientation has been identified as fundamental to integration of IENs into the clinical area. ${ }^{[21]}$ Nurses need to become more familiar with IEN program and course expectations so there is increased understanding of staff (as educator) and student role in the setting. Orientation about the bridging program and the IENs experiences including credentialing could be done previous to IENs arriving on the unit. The utilization of focused clinical units within an organization with consistent clinical faculty would help to establish and improve integration of the IENs into the clinical area.

In relation to the theme insider-outsider, the IENs more often felt as outsiders. Being accepted on the unit is a critical component of being integrated into the clinical setting. Jose ${ }^{[22]}$ found that IENs regarded support from co-workers as vital for adaptation to and survival in the workplace. To improve the IENs' integration, nurses and clinical instructors need to view culture from a constructivist perspective instead of from a traditional essentialist view. Thus, nurses and teachers can gain a greater understanding about how culture shapes, but does not define people; culture is dynamic - ever changing; culture is individual in nature; and culture is related to historical, political, and social contexts. Diversity education for all nurses in an organization would benefit the work environment of the IEN but also benefit the care environment for the patient and families. Hagey et al. ${ }^{[23]}$ suggested that all nurses in all levels of the profession need to become educated about diversity and to learn about discriminatory practices.
Wheeler, Foster and Hepburn ${ }^{[24]}$ recommended that healthcare institutions ensure policies are in place for addressing discrimination, and supervisors may require more training to support nurses and manage discriminatory interactions.

IENs shared their experiences where they were viewed as an outsider by the patients. The IENs expressed feeling that they were being judged by their skin color and the patients did not respect them or view them as the qualified nurse. The IENs experienced racism. $\mathrm{Xu}^{[6]}$ identified that there is widespread racism against IENs and that bridging programs have not always addressed fundamental social issues as racism, discrimination and interpersonal conflicts. ${ }^{[10]}$ In the pan-Canadian Framework of Guiding Principles and Essential Components for IEN Bridging Programs, ${ }^{[25]}$ providing a safe learning environment with culturally competent faculty has been identified as one of the guiding principles for all bridging programs. Nurse managers have a role in incorporating a professional culture including value of diversity in a nursing unit as well as throughout the organization. ${ }^{[21]}$ In a study with IENs by Hagey et al., ${ }^{[23]}$ participants in the study viewed breaking the silence about racism in nursing as a fundamental duty. By not doing so, the not so subtle message being delivered is acceptance of or lack of motivation and leadership to address discriminatory behaviours in the practice setting.

In the theme of comparing and contrasting, IENs compared the Canadian health care system with the health care system in their country of origin. The difference in scopes of practice for the nurse involved more responsibility in educating the patients, working with different team members and in organizing and providing holistic care including discharge planning. IENs spoke about aspects of the nurse's role in Canada which would be completed by other members of the health care team in their country of origin. Nurses in Canada performed roles that physicians in other countries would complete, for example with discharge planning. IENs would have a large nurse-to-patient assignment and work with personal support workers in expanded roles. Health care delivery systems in other countries include large numbers of patient care assistants. ${ }^{[26]}$ IENs were required to adapt to the new roles and differences in roles in the health care system. In a study by Tregunno et al. ${ }^{[11]}$ IENs identified differences in practice in Canada involving more responsibility for patients, more involvement in clinical decision making, respected more by other disciplines and less hierarchy with physicians as compared to their country of origin.

Challenges for the IEN included dis-continuity, technology, and communication. Dis-continuity involved the lack of continuity when working with the nursing staff due to the 
frequent change in nursing staff. The constant change in staff prevents the IEN from developing confidence in understanding expectations when working with different mentors and the constant change prevents the IEN from developing a support system on the unit. One IEN identified that the change in staff also affects patient care because there is much time consumed in finding out the history of the patient's hospital stay. Continuity in patient care has been found to positively influence health outcomes. ${ }^{[27,28]}$ Similarly, positive learning outcomes could result when students are consistently working with a small team of nurses so that collegial relationship building and trust has a chance to be established.

The use of new and different technologies has been identified as a challenge to integration by the IEN. Challenges with technology have also been identified by Jose. ${ }^{[22]}$ IENs were pleased with learning new technologies but required more time, education and support to master the technologies. IENs felt that they would require extensive assistance with technology on entering practice. ${ }^{[29]}$ The academic and practice setting could partner to invest more of a focus on preparing IENs for the types of technologies that will be encountered in hospital settings. A theory-based course related to informatics would also extend IENs' knowledge regarding health literacy for themselves and for patients. The Canadian Association of Schools of Nursing (CASN) has defined entry to practice informatics competencies that can be integrated into the curriculum for IENs to provide knowledge and skills to practice in an increasingly technological-enabled environment. ${ }^{[30]}$ Communication was seen as a major challenge when speaking with patients, in documentation, understanding and using short forms and abbreviations, using the telephone and when taping and listening to report. Documenting findings independently has also been seen as a challenge by other IENs. ${ }^{[29]}$ Jose $^{[22]}$ reported that for the IENs who were used to receiving only written orders from doctors that taking verbal and telephone orders could be very distressing. Additional research has shown that communication is a major challenge to workplace integration for IENs. ${ }^{[7,11,31]}$ A focus on workplace communication would benefit IENs. Education programs for IENs may need to review communication courses to include more opportunities to improve language skills. For instance, the CARE (Creating Access to Regulated Employment) Centre for Internationally Educated Nurses has developed workplace communication courses that are specific to meet IENs' needs. ${ }^{[32]}$ The three level courses include therapeutic communication, nurse-client interviews, documentation and reporting, assertiveness and working with the interprofessional team. ${ }^{[32]}$

For the final theme, IENs were often seeking opportunities for learning. The IENs were eager to learn in order to be more knowledgeable and better prepared as nurses. They wanted to take more courses, read the policies and procedures of the agency and be prepared to participate in the care of the patient. In a study on IENs transitioning into the workplace, Sherman and Eggenberger ${ }^{[26]}$ reported that nursing leaders found international nurses in their work areas to be extremely receptive to education and learning. The IENs were viewed as smart, willing to learn, loyal and hard working. IENs have identified the importance of education about hospital policies and procedures. ${ }^{[22]}$ IENs are often very enthusiastic to become well integrated members of a clinical unit.

\section{Limitations}

A limitation of the study relates to the over-representation of females in the sample. However, the inclusion of one male participant reflected the general composition of the educational program in which IENs were enrolled. The study findings are limited to the acute care setting where the participants were engaged in clinical practice as IENs.

\section{Conclusion}

This study furthers the understanding of IENs' integration into the clinical setting. Based on the themes identified, IEN students are encouraged to discuss with clinical teachers about the challenges they might encounter with clients or staff nurses. IENs need to develop enhanced conflict management skills to be able to more effectively engage in critical conversations with nursing staff and faculty about client care or colleague situations. Additional research is required to pursue understanding of IEN experiences of integration in a variety of practice settings. Further, research with staff nurses and clinical instructors is warranted to gain an understanding of their perspective of integration of IENs during practice-based experiences, as such knowledge could provide a broader understanding IEN education and serve to provide additional strategies for improvement in the process of integration. Development of a specific unit as a model unit for integration of IENs in any setting would benefit both the staff and IENs on the unit. Finally, cultural education of all unit employees would enhance the understanding and collaboration among the team members.

\section{ACKNOWLEDgements}

This research was funded by Fanshawe College Research Innovation Fund.

\section{CONFLicts OF INTEREST Disclosure}

The authors declare that there is no conflict of interest statement. 


\section{REFERENCES}

[1] Canadian Nurses Association [CNA]. Tested solutions for eliminating Canada's Registered Nurse shortage. 2009. Available from: http://www.cna-aiic.ca/sitecore $\% 20$ modules/web/ /me $\mathrm{dia} / \mathrm{cna} /$ page $\% 20$ content/pdf $\% 20$ en/2013/07/26/10/41/r n_highlights_e.pdf

[2] Staiger DO, Auerbach DI, Buerhaus PI. Registered Nurse labor supply and the recession-are we in a bubble? New England Journal of Medicine. 2012; 366(16): 1463-1465. Available from: http://www.dartmouth.edu/ dstaiger/Papers/201 2/staiger $\% 20$ et $\% 20$ al $\% 20$ bubble $\% 20$ ne jm $\% 202012$.pdf

[3] Buchan J, Seccombe I. Overstretched. Under-resourced. The UK nursing labour market review. 2012. Royal College of Nursing. 2013 Available from: http://www.rcn.org.uk/__data/assets/pdf _file/0016/482200/004332.pdf

[4] Horne G. Canada's policy framework for the utilization of internationally educated nurses. Queen's Policy Review. 2011; 2(2): 37-53. Available from: http://www. queensu.ca/sps/qpr/issues/vo 12issue2/Horne.pdf

[5] Alexis O. Internationally educated nurses' experiences in a hospital in England: An exploratory study. Scandinavian Journal of Caring Sciences. 2013; 27: 962-968. http://dx.doi.org/10.1111/scs .12014

[6] Xu Y. Strangers in strange lands. A meta-synthesis of lived experiences of immigrant Asian nurses working in Western countries. Advances in Nursing Science. 2007; 30(3): 246-265. PMid:17703124 ht tp://dx.doi.org/10.1097/01.ANS.0000286623.84763.e0

[7] Blythe J, Baumann A, Rheaume A, et al. Nurse migration to Canada. Pathways and pitfalls of workforce integration. Journal of Transcultural Nursing. 2009; 20(2): 202-210. http://dx.doi.org/10.11 $77 / 1043659608330349$

[8] Blythe J, Baumann A. Supply of internationally educated nurses in Ontario: Recent developments and future scenarios. Health Human Resources Series Number 9. Nursing Health Services Research Unit, McMaster University. 2008. Available from: http: //www . healthworkermigration.com/newsevents/262-sup ply-of-internationally-educated-nurses-in-ontario -recent-developments-and-future-scenarios.html

[9] Bourgeault IL, Neiterman E, LeBrun J, et al. Brain gain, drain \& waste: The experiences of internationally educated health professionals in Canada. University of Ottawa, 2010. Available from: http://www.threesource.ca/documents/February2 011/brain_drain.pdf

[10] Zizzo KA, Xu Y. Post-hire transitional programs for international nurses: A systematic review. The Journal of Continuing Education in Nursing. 2009; 40(2): 57-66. http://dx.doi.org/10.3928/002 20124-20090201-02

[11] Tregunno D, Peters S, Campbell H, et al. International nurse migration: U-turn for safe workplace transition. Nursing Inquiry. 2009; 16(3): 182-190. http://dx.doi.org/10.1111/j.1440-1800. 2009.00448.x

[12] Canadian Institute of Health Information [CIHI]. Regulated nurses Canadian trends 2007-2011. 2013. Available from: https://secu re.cihi . ca/estore/productFamily.htm?pf =PFC2016

[13] College of Nurses of Ontario. Membership statistics highlights 2013 2013. Available from: http://www.cno.org/Global/docs/ge neral/43069_stats/43069_MembershipStatistics-Highl ights.pdf?utm_source=cno.org\&utm_medium=link_in_pa ge\&utm_term=MembershipHighlightsPDF2013\&utm_campa ign=MembershipHighlightsPDF20139

[14] Westra BL, Rodgers BL. The concept of integration: A foundation for evaluating outcomes of nursing care. Journal of Professional Nursing.
1991; 7(5): 277-282. http://dx.doi.org/10.1016/8755-722 3 (91) 90114-Z

[15] Heidegger M. Being and time. (Mac Quarrie, J. \& Robinson, E. trans). 1962. Harper and Row. New York. In Van Der Zalm, J.E. \& Bergum, V. Hermenutic-phenomenology. Providing living knowledge for nursing practice. Journal of Advanced Nursing. 2000; 31(1): 211-218. http://dx.doi.org/10.1046/j.1365-2648.2000.01244.x

[16] Van Manen M. Researching lived experience: Human science for an action sensitive pedagogy. London, Ontario: Althouse, 1994.

[17] Sadala MLA, Adorono RDF. Phenomenology as a method to investigate the experience lived: A perspective from Husserl and Merleau Ponty's thought. Journal of Advanced Nursing. 2002; 37(3): 282-293. http://dx.doi.org/10.1046/j.1365-2648.2002.02071.x

[18] Van Der Zalm JE, Bergum V. Hermeneutic-phenomenology. Providing living knowledge for nursing practice. Journal of Advanced Nursing. 2000; 31(1): 211-218. PMid:10632811 http://dx.doi . org/10.1046/j.1365-2648.2000.01244.x

[19] Smith JA, Osborn M. Interpretive phenomenological analysis. In J. A. Smith, Qualitative psychology: A practical guide to research methods. Thousand Oaks, CA: Sage, 2007. Available from: http: //www . sagepub.com/upm-data/17418_04_Smith_2e_Ch_04.pdf

[20] Van Manen M. Practicing phenomenological writing. Phenomenology and Pedagogy. 1984; 2(1): 36-69.

[21] Higginbottom GMA. The transitioning experiences of internationallyeducated nurses into a Canadian healthcare system: A focused ethnography. BMC Nursing. 2011; 10: 14. PMid:21693059 http: //dx.doi.org/10.1186/1472-6955-10-14

[22] Jose MM. Lived experiences of internationally educated nurses in hospitals in the United States of America. International Nursing Review. 2011; 58(1): 123-129. PMid:21281304 http://dx.doi.org /10.1111/j.1466-7657.2010.00838.x

[23] Hagey R, Choudhry U, Guruge S, et al. Immigrant nurses' experience of racism. Journal of Nursing Scholarship. 2001; 33(4): 389394. PMid:11775311 http://dx.doi.org/10.1111/j.1547-5 $069.2001 .00389 . x$

[24] Wheeler RM, Foster JW, Hepburn KW. The experience of discrimination by US and internationally educated nurses in hospital practice in the USA: A qualitative study. Journal of Advanced Nursing. 2014; 70(2): 350-359. PMid:23782320 http://dx.doi.org/10.1111 /jan. 12197

[25] Canadian Association of Schools of Nursing [CASN]. Pan-Canadian framework of guiding principles \& essential components for IEN bridging programs. Ottawa, ON: CASN, 2012.

[26] Sherman R, Eggenberger T. Transitioning internationally recruited nurses into clinical settings. The Journal of Continuing Education in Nursing. 2008; 39(12): 535-546. http://dx.doi.org/10.3928 /00220124-20081201-03

[27] Bjorkelund C, Maun A, Murante AM, et al. Impact of continuity on quality of primary care: From the perspective of citizens' preferences and multimorbidity-position paper of the European Forum for Primary Care. Quality in Primary Care. 2013; 21(3); 193-204. Available from: http://web.b.ebscohost.com.proxy1.lib.uwo .ca/ehost/pdfviewer/pdfviewer?vid=3\&sid=c41a99d0-f 1e6-4844-96e5-293e48d9df58\%40sessionmgr 198\&hid=103

[28] Havens DS, Vasey J, Gittell JH, et al. Relational coordination among nurses and other providers: impact on the quality of patient care Journal of Nursing Management. 2010; 18(8): 926-937.

[29] Edwards PA, Davis CR. Internationally educated nurses' perceptions of their clinical competence. Journal of Continuing Education in Nursing. 2006; 37(6): 265-269. Available from: http://search.proquest.com.proxy1.lib.uwo.ca/nurs 
ing/docview/223324616/fulltext/2DBF67BF597E4C7CPQ/ 12 ? accountid $=15115$

[30] Canadian Association of Schools of Nursing [CASN]. Nursing informatics. Entry-to-practice competencies for Registered Nurses. Ottawa, ON: CASN, 2012.

[31] Thekdi P, Wilson BL, Xu Y. Understanding post-hire transitional challenges of foreign-educated nurses. Nursing Management. 2011; 42(9): 8-14. http://dx.doi.org/10.1097/01. NUMA.0000403 285.34873.c7

[32] CARE. Centre for Internationally Educated Nurses. CARE language courses. Toronto, ON; CARE, 2014. Available from: http://www . care4nurses.org/what-we-do/language/\#ql-courses 